\title{
Vortex tracking in high density vector fields
}

\author{
Clément Guérin ${ }^{1}$, Petra Gomez-Krämer ${ }^{1}$, Michel Ménard ${ }^{1}$, and Damien \\ Coisne $^{2}$ \\ ${ }^{1}$ L3I, University of La Rochelle, France ${ }^{2}$ University Hospital of Poitiers, France \\ \{clement.guerin, petra.gomez, michel.menard\}@univ-lr.fr \\ \{damien.coisne\}@chu-poitiers.fr
}

\begin{abstract}
Echo Particular Velocity Imaging (EchoPIV) has become a promising tool to detect flow patterns in cardiovascular structures. We present in this paper a new method for the detection and tracking of the vortex in the left ventricle for EchoPIV image sequences. The vortex is roughly localized by approximating an affine motion model to the estimated high density motion vector field and analyzing the significance of its rotational component in a statistical test. Based on a detection of the center of rotation and the approximated motion model, the area of the vortex is segmented. False positives are eliminated by analyzing the vortex's shape. Results on synthetic and real data are promising.
\end{abstract}

Keywords: Vortex detection/tracking, motion analysis, blood flow, EchoPIV

\section{Introduction}

The analysis of fluid motion is often an essential task for interpreting results in several application domains [1-3]. The issue in medical imaging is to visualize and analyze the blood flow inside the heart, or inside blood vessels.

Echo Particular Velocity Imaging (EchoPIV) has become a promising tool to detect flow patterns in cardiovascular structures. It has been introduced as a new ultrasound methodology to measure 2D intra-ventricular flow patterns. Based on such EchoPIV sequences of the left ventricle, our objective is to analyze automatically the behavior of the incoming blood flow which can vary depending on the age and the health of the patient, and the shape of his heart. The most visible pattern in those images is a vortex that occurs after the opening of the mitral valve and disappears at the opening of the aortic valve. The parameters of this vortex like its size, velocity, or position of the center are today unfamiliar to clinicians because there has been no tool until EchoPIV to visualize such information.

In order to detect, track, and analyze the vortex in the left ventricular cavity, we estimate a set of motion vector fields representing the instantaneous motion of the blood flow. We analyze those motion vector fields, looking for singularities, namely the dominant rotational motion of the vortex. The aim is to determine if they contain a curl area that corresponds to a vortex, and to evaluate its size and center of rotation which is used for tracking. 

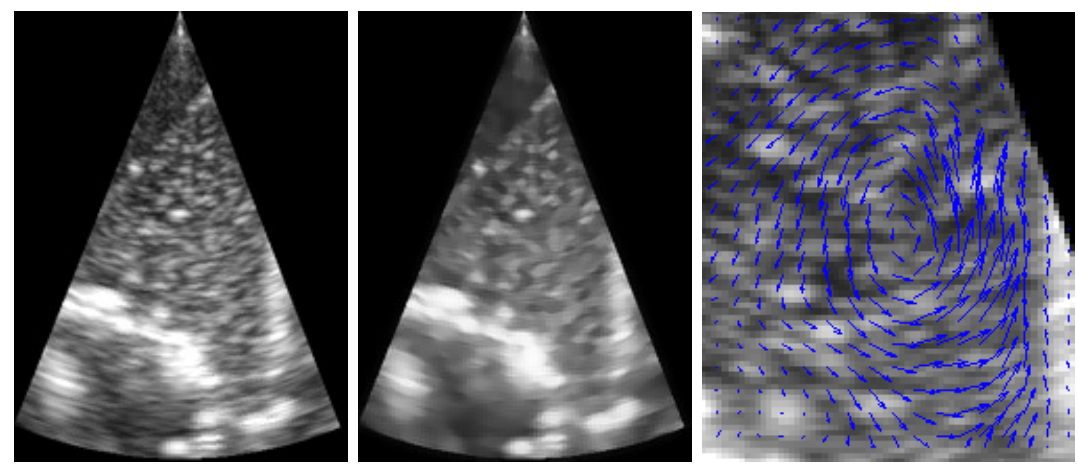

Fig. 1. Left: EchoPIV image of the left ventricle of an artificial heart, middle: geometrical component, right: vortex with irregular shape and vector repartition.

The reminder of this paper is organized as follows: We present briefly in Section 2 the acquisition of cardiographic EchoPIV sequences and the estimation of the motion vector fields. In Section 3 we present our analysis method of the motion vector field to detect, track, and extract information on the vortex. We show and discuss some results in Section 4 and conclude our work in Section 5.

\section{Motion estimation for EchoPIV image sequences}

EchoPIV combines the advantages of PIV and ultrasound for the robust and efficient analysis of blood flow. Indeed, it is a contrast-enhanced ultrasound method where the evolution of micro bubbles of an inert gas, injected into the blood stream, are tracked. This method, first introduced in the nineties, has the advantage that is not dangerous for the patient as the gas bubbles dissolve. Moreover, the exam is not invasive, less costly, and needs only a few minutes to acquire the image sequence. For the analysis of the blood flow in the left ventricle, we acquire a sequence of 180 frames per second with a resolution of $108 \times 80$ pixels during four heartbeats on an artificial heart.

The major disadvantage of the EchoPIV is that the images are very noisy and have low resolution, which are difficult to analyze without preprocessing (see Fig. 1). Moreover, the bubbles entering and leaving the acquisition plane add some difficulty to the motion estimation. Those inter-plane motions create a turbulent and changing dynamic texture, which is not characterized by global motion. In order to obtain a robust and reliable motion estimation, an extended Meyer decomposition [4] is applied, which decomposes the image into three components, containing geometrical, textural, and noise information. Based on the geometrical component, the optical flow is estimated using a partial derivative equation (Lucas Kanade), and mean (4 cycles) flow velocity fields were computed $[5]$. 


\section{$3 \quad$ Vortex detection and tracking}

The vortex detection and tracking process is composed of four main steps for each image.We first approximate a $2 \mathrm{D}$ affine motion model to the motion vector field that is analyzed to detect the area with most significant rotational motion, which is assumed to be a part of the vortex. Then, the center of the rotation is estimated that is used in a segmentation step in order obtain the exact area of the vortex. Afterwards, we analyze the shape of the detected vortex to eliminate false positives. The vortex's center is then tracked through the sequence.

\subsection{Detection of regions with significant rotation}

In order to approximate the motion of singularities, it is common to use an affine 2D motion model of the apparent motion. It is defined as:

$$
\mathbf{d}=\mathbf{A} \boldsymbol{x}+\boldsymbol{t}
$$

where $\mathbf{A}=\left(\begin{array}{cc}a_{1} & a_{2} \\ a_{3} & a_{4}\end{array}\right)$ is a matrix describing the linear motion, and $\boldsymbol{t}=\left(t_{x}, t_{y}\right)^{\mathrm{T}}$ is a vector describing the translational motion, $\boldsymbol{x}=(x, y)^{\mathrm{T}}$ are the image centered coordinates, and $\mathbf{d}=\left(d_{x}, d_{y}\right)^{\mathrm{T}}$ is the motion vector. Thus, the motion is determined through the knowledge of the matrix A and the vector $\boldsymbol{t}$.

Several authors have proposed to analyse the matrix $\mathbf{A}$ to detect singularities in the flow pattern e.g. [3]. However, the analysis of the motion parameters is difficult and less robust in case of complex and noisy motion. Moreover, we are in particular interested in regions with a dominant rotational component that is assumed to be a part of the vortex. For this reason, we use a statistical test based on $[6,7]$ in order to determine whether the rotational component is significant in a certain region or not.

We use the representation of [6] of the affine motion model to characterize dominant blood motion in a block $B$ of size $M \times M$. This representation is more related to the physical meaning and more convenient for the interpretation of the dominant motion. The affine model (1) can be expressed as:

$$
\mathbf{d}(\mathbf{p}, \phi)=\left\{\begin{array}{l}
d_{x}=t_{x}+\operatorname{div} \cdot x-\operatorname{rot} \cdot y+h y p 1 \cdot x+h y p 2 \cdot y \\
d_{y}=t_{y}+\operatorname{div} \cdot y+\operatorname{rot} \cdot x-h y p 1 \cdot y+h y p 2 \cdot x
\end{array}\right.
$$

with div $=\frac{1}{2}\left(a_{1}+a_{4}\right)$, rot $=\frac{1}{2}\left(a_{3}-a_{2}\right)$, hyp $1=\frac{1}{2}\left(a_{1}-a_{4}\right)$, and hyp $2=\frac{1}{2}\left(a_{2}+a_{3}\right)$.

The linear model $(2)$ can be written in a general matrix form:

$$
\mathbf{z}=\mathbf{H} \phi+\mathbf{v} \quad \text { with } \mathbf{H}=\left(\begin{array}{cccccc}
1 & 0 & x_{1} & -y_{1} & x_{1} & y_{1} \\
1 & 0 & x_{N} & -y_{N} & x_{N} & y_{N} \\
0 & 1 & y_{1} & x_{1} & -y_{1} & x_{1} \\
0 & 1 & y_{N} & \dddot{x}_{N} & -y_{N} & x_{N}
\end{array}\right)
$$

where $\mathbf{z}$ is the vector containing the motion vectors from Section $2, \mathbf{H}$ is the observation matrix containing the $N=M \cdot M$ image centered coordinates of the block $B, \phi=\left(t_{x}, t_{y} \text {, div, rot, hyp } 1, \text { hyp } 2\right)^{\mathrm{T}}$ is the vector of the motion parameters, and $\mathbf{v}$ is the vector of measurement noise. 
The vector of the motion parameters $\hat{\phi}$ can then be estimated by a least square estimation as:

$$
\hat{\phi}=\left(\mathbf{H}^{\mathrm{T}} \mathbf{H}\right)^{-1} \mathbf{H}^{\mathrm{T}} \mathbf{z}
$$

In order to determine if the rotational component rot of the estimated model $\hat{\phi}$ is significant or not we perform a statistical test considering two hypotheses. The first hypothesis $H_{0}$ assumes that the rotational component is significant. The second one $H_{1}$ assumes that it is not significant i.e. it equals zero, while the other five parameters are free and have to be reestimated. $\hat{\phi}_{0}$ and $\hat{\phi}_{1}$ are the motion models corresponding to the hypotheses $H_{0}$ and $H_{1}$.

The likelihood function $f$ for each hypothesis is defined with respect to the vector of residuals $\mathbf{r}\left(\hat{\phi}_{H}\right)=\mathbf{d}-\mathbf{d}\left(\hat{\phi}_{H}\right)$ with $\mathbf{d}$ the original motion vector of Section 2 and $\mathbf{d}\left(\hat{\phi}_{H}\right)$ the motion vector conform to $\hat{\phi}_{H}, H=0,1$. The residuals are supposed to be independent and to follow a zero-mean Gaussian law:

$$
f\left(\mathbf{r}\left(\hat{\phi}_{H}\right)\right)=\prod_{i \in B}\left(\frac{1}{2 \pi \sqrt{\operatorname{det}\left(\Sigma_{H}\right)}} \exp ^{-\frac{1}{2}\left(\mathbf{r}_{i}\left(\hat{\phi}_{H}\right)^{T} \Sigma_{H}^{-1} \mathbf{r}_{i}\left(\hat{\phi}_{H}\right)\right)}\right)
$$

with $\Sigma_{H}=\left(\begin{array}{c}\sigma_{x, H}^{2} 0 \\ 0 \\ \sigma_{y, H}^{2}\end{array}\right)$ as the covariance matrix and $\sigma_{x, H}^{2}$ and $\sigma_{y, H}^{2}$ the variances of the $\mathrm{x}$ - and $\mathrm{y}$-components of the residuals. In practice, $\hat{\phi}_{1}$ is obtained by (4) and we use a least square estimation similarly to (4) to estimate the vector $\hat{\phi}_{1}$. In order to set the rotational component to zero, we use a reduced parameter vector $\bar{\phi}=\left(t_{x}, t_{y} \text {, div, hyp } 1, \text { hyp } 2\right)^{\mathrm{T}}$ and a reduced observation matrix $\overline{\mathbf{H}}$ where the fourth column is suppressed. Then, the ratio $s$ is called the significance value:

$$
s=\ln \left(\frac{f\left(\mathbf{r}\left(\hat{\phi}_{1}\right)\right)}{f\left(\mathbf{r}\left(\hat{\phi}_{0}\right)\right)}\right)=N \cdot\left(\ln \left(\sigma_{x, 1} \cdot \sigma_{y, 1}\right)-\ln \left(\sigma_{x, 0} \cdot \sigma_{y, 0}\right)\right)
$$

The higher the value $s$, the more significant is the rotational component of a block. The block with the highest rotational component is considered to be a part of the vortex. It is used in the next step for the rotation center detection.

\subsection{Rotation center estimation}

The vortex segmentation step of Section 3.3 assumes the knowledge of the vortex's rotation center. To this end, we use the method introduced by [8] as a basis, which consists in literally making the vectors turn around their base to point to the rotation center. In contrast to [8], we determine the angle $\alpha$ for which each vector has to be rotated depending on the approximated model $\hat{\phi}$ of Eq. (4):

$$
\alpha=\operatorname{atan} 2(\phi(4),-\phi(3))
$$

Then, each rotated vector is expanded to a sector with span $\sigma$ similarly to [8]. The span $\sigma$ is fixed to $\pi / 6$ in our case. At least, the region covered by the largest number of sectors within a distance of twice the block size is the rotation center. Fig. 2 shows an example of a noised synthetic spiral (zoom + dominant rotation) and the detected center. 

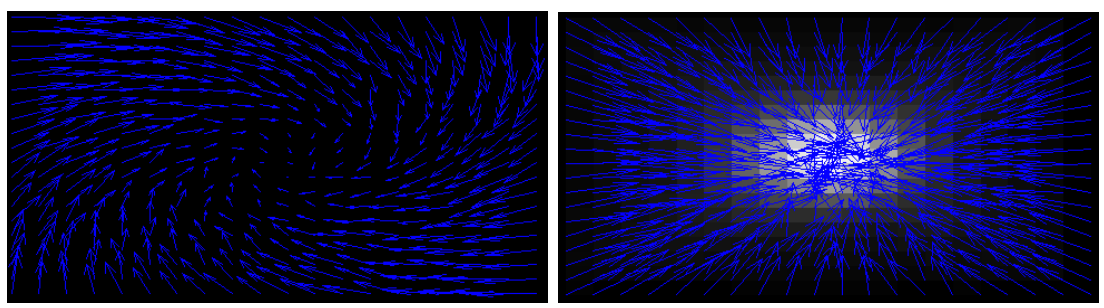

Fig. 2. Left: noised spiral, right: rotated vectors pointing to the center. The brightest pixel represents the identified center.

\subsection{Vortex segmentation}

The objective is now to segment the area of the vortex using its rotation center. Therefore, we refine the parameter vector $\hat{\phi}$ by Eq. (4) on a block which is centered on the rotation center. Using $\hat{\phi}$ we calculate the synthetic pattern of the vortex. The idea is to compare those synthetic vectors $\hat{\mathbf{d}}$ with the real ones d to specify an area around the rotation centre, where the motion is consistent with the estimated parameters $\hat{\phi}$.

To decide whether a vector $\mathbf{d}$ should be associated to the segmented vortex or not, we compute its similarity to the vector $\hat{\mathbf{d}}$ in the synthetic pattern. Our similarity measure takes account of the difference in size and angle of both motion vectors, as well as the distance from the rotation center:

$$
\begin{aligned}
& S=S_{N} S_{D} W \quad S_{N}=\left|\frac{\min (\|\mathbf{d}\|,\|\hat{\mathbf{d}}\|)}{\max (\|\mathbf{d}\|,\|\hat{\mathbf{d}}\|)}\right| \quad W=\frac{K}{\sqrt{\left(x_{r}-x\right)^{2}+\left(y_{r}-y\right)^{2}}} \\
& S_{D}=1-\left|\left(\frac{\min \left[\left(\alpha_{\mathbf{d}}-\alpha_{\hat{\mathbf{d}}}\right) \bmod 2 \pi,\left(\alpha_{\mathbf{d}}-\alpha_{\hat{\mathbf{d}}}\right)\right.}{\bmod 2 \pi]}\right)\right|
\end{aligned}
$$

where $\alpha_{\mathbf{d}}$ and $\alpha_{\hat{\mathbf{d}}}$ are the angles of the motion vectors $\mathbf{d}$ and $\hat{\mathbf{d}}$ with the $\mathrm{x}$-axis. $\left(x_{r}, y_{r}\right)$ are the coordinates of the rotation center and $(x, y)$ the coordinates of the motion vector. The constant $K$ influences the slope of the decaying weighting function and is empirically set to $10 \mathrm{in}$ our experiments. The closer $S_{N}$ and $S_{D}$ to 1 , the more similar are the vectors. When $S$ exceeds a threshold $T_{1}$ the vector $\mathbf{d}$ is associated to the segmented vortex. $T_{1}$ equals 0.3 in our experiments.

\subsection{False positive elimination}

A blood vortex occurs and dissolves at some specific instant of the cardiac cycle. This means that there are several images in the sequence that do not contain any vortex or contain some half rotation at the moments when the vortex occurs or dissolves. Thus, we have to determine if we actually deal with a vortex or not. The incoming flow from the mitral valve often provokes a north-directed flow as shown in Fig. 1, which is often associated to vortex in the segmentation 
step. Hence, we cannot rely our analysis on the symmetry of vortex nor an equal distribution of motion vector angles because of the irregular shape of the segmented pattern and the irregular repartition of motion vectors.

The idea here is to compare our vortex with a pure rotation to reject some partial rotational patterns. We consider the bounding box of the segmented area to recalculate its synthetic motion vectors using only the rotational parameter $\phi=(0,0,0, r o t, 0,0)^{\mathrm{T}}$. For reestimating the rotational parameter by Eq. (4) we suppress all columns except the fourth one in the matrix $\mathbf{H}$ in order to set the other parameters to 0 in the estimation. Then, we compare each vector of the segmented vortex to its synthetic match in the pure rotational pattern, according to Eq. (8). At least, if the ratio of matching/unmatching vectors in the bounding box is higher than a predefined threshold $T_{2}$, we consider that we actually deal with a vortex. $T_{2}$ is set to 0.7 in our experiments.

\section{Results}

We present in this section some results obtained on synthetic and real data. As we want to evaluate the performance of our algorithm, we created a set of 50 motion vector fields $(108 \times 80$ vectors $)$ where 28 contain a vortex according to the motion parameters $\phi=\{0,0,[-1 ;-0.5],[0.5 ; 1], 0,0\}$. The others contain only half rotations. The size and position of each vortex pattern were randomly generated and random noise was added afterwards. With the proposed method, we obtained $71 \%$ and $90 \%$ of precision and recall of vectors correctly segmented as a part of the vortex. We generated a second set of 3000 motion vector fields where the motion parameters, the influence of the added noise, the noisiness, size, and position of the vortex vary. We obtained $81 \%$ of correctly detected vortices, $99 \%$ precision and $86 \%$ recall of correctly segmented vectors, and a mean error of 0.17 pixels for the vortex center detection.

We used in our experiments a block size of $17 \times 17$ pixels. On the one hand, the choice of the block size for significant rotation detection (Section 3.1) depends on the size of the vortex to be detected. If it is too small, a singularity or noise in the optical flow causes a high rotational component and so probably a vortex detection for only few vectors. If it is too large, the precision of vortex localization drops as the rotational motion cannot be distinguished from the other motions in the block. On the other hand, the precision of the rotation center estimation (Section 3.2) increases with the number of used motion vectors.

Fig. 3 presents some results obtained on an EchoPIV sequence of an artificial heart. Some estimated motion vector fields and the result of the vortex detection are shown at different moments of the cardiac cycle. We can observe the occurrence of the vortex after the opening of the mitral valve and its dissolution at the opening of the aortic valve. The evolution of the vortex's size and rotation through the cardiac cycle are visualized in Fig. 4. The graphs are quite noisy and some misdetections can be observed, which nevertheless allows to interpret the result. The size of the vortex increases slowly after its occurrence (image no. 13) and decreases rapidly at its dissolution (no. 127-148). The rotation of 


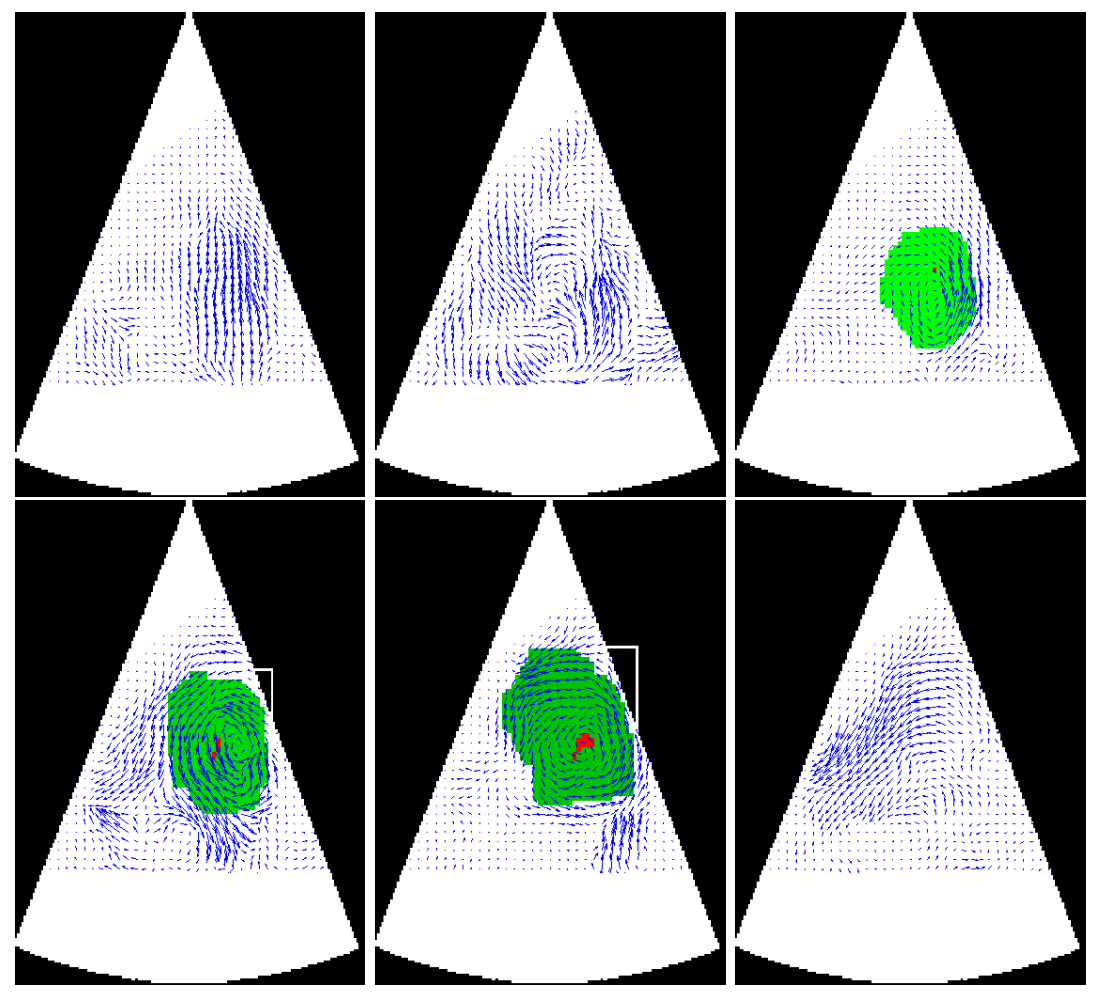

Fig. 3. From top left to bottom right: evolution of the cardiac cycle. The darker the green of the vortex the stronger is its rotational component. The red dots show the evolution of the center position through the cardiac cycle.

the vortex accelerates at its occurrence (no. 13-31) and decreases slowly until its dissolution (no. 148). Results on EchoPIV images depend strongly on the quality of the optical flow. Tests using Horn and Schunk optical flow have shown inconsistent vortex detection as, due to the strong noise, it does not represent well the flow pattern.

Although our algorithm is quite fast, it takes approximatively $0.2 \mathrm{~s}$ per motion vector field on a $2.4 \mathrm{GHz}$ Core 2 Duo processor running on Mac OS X, it is not yet meant to be applied in real time. The estimation of the motion vector fields needs a frame rate above 180 frames per second to be accurate and is quite time consuming. As the significant rotation detection consists of an exhaustive evaluation over the image, we choose a step size of 4 pixels for moving the window to reduce computational costs. However, it works well on a posteriori data and permits to track the vortex through the cardiac cycle and to monitor the evolution of its size, position etc. Furthermore, the implementation of our algorithm in MATLAB is not optimized. Hence, computational times can be still be reduced. 

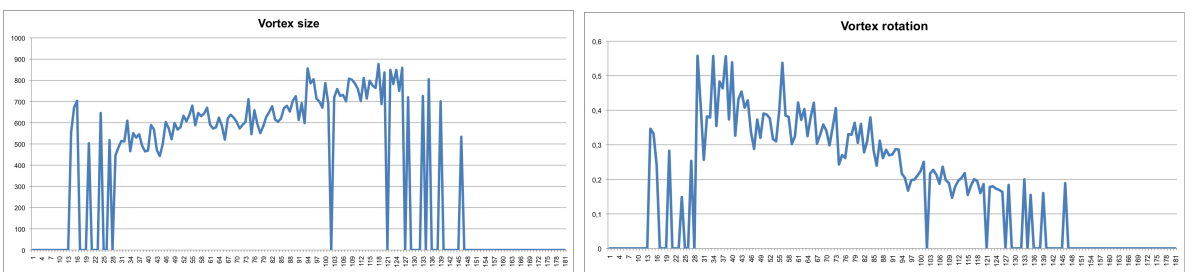

Fig. 4. The evolution of the vortex through the cardiac cycle. Left: size, right: rotation.

\section{Conclusion}

We presented a complete method to detect, track, and analyze the blood vortex through a cardiac cycle in the left ventricle. The vortex is assumed to contain a dominant rotational motion. Therefore, our method is based on the approximation of an affine motion model and the analysis of the significance of its rotational component. The results on synthetic data are promising. Further results were presented for a real EchoPIV sequence of an artificial heart. The detected vortex was analyzed in terms of size and rotation speed. The next step of our work will be the evaluation of our method in clinical trials and to improve the vortex detection rate.

\section{References}

1. S. Song and R.M. Leahy. Computation of 3D velocity fields from $3 \mathrm{D}$ cine CT images of a human heart. IEEE Trans. Med. Imaging, MI-10:295-306, 1991.

2. T. Corpetti, E. Mémin, and P. Pérez. Dense motion analysis in fluid imagery. In ECCV, LNCS 2350, pages 676-691, 2002.

3. C.-F. Shu and R.C. Jain. Direct estimation and error analysis for oriented patterns. CVGIP: Image Underst., 58:383-398, 1993.

4. A. El Hamidi, M. Ménard, M. Lugiez, and C. Ghannam. Weighted and extended total variation for image restoration and decomposition. Pattern Recognit., 43(4):1564-1576, 2010.

5. D. Coisne, C. Guérin, M. Lugiez, M. Ménard, C. Guivier-Curien, D. Tann, and L. Christiaens. Validation against laser PIV of a new algorithm of echo contrast tracking based on optical flow algorithm for ECHO PIV method. Circulation, 122, 2010.

6. P. Bouthemy, M. Gelgon, and F. Ganansia. A unified approach to shot change detection and camera motion characterization. IEEE Trans. Circuits Syst. Video Technol., 9(7):1030-1044, 1999.

7. P. Krämer and J. Benois-Pineau. Camera motion detection in the rough indexing paradigm. In TRECVID Online Proceedings, 2005.

8. K.Y. Wong and C.L. Yip. Identifying centers of circulating and spiraling vector field patterns and its applications. Pattern Recognit., 42(7):1371-1387, 2009. 